\title{
COVID-19, the Yule-Simpson paradox and research evaluation
}

\author{
Zhiqi Wang $^{1} \cdot$ Ronald Rousseau ${ }^{2,3}$ (I)
}

Received: 22 September 2020 / Published online: 7 February 2021

(c) Akadémiai Kiadó, Budapest, Hungary 2021

\begin{abstract}
The Yule-Simpson paradox refers to the fact that outcomes of comparisons between groups are reversed when groups are combined. Using Essential Sciences Indicators, a part of InCites (Clarivate), data for countries, it is shown that although the Yule-Simpson phenomenon in citation analysis and research evaluation is not common, it isn't extremely rare either. The Yule-Simpson paradox is a phenomenon one should be aware of, otherwise one may encounter unforeseen surprises in scientometric studies.
\end{abstract}

Keywords Yule-Simpson paradox $\cdot$ Relative citations $\cdot$ Scientometric comparisons between countries

\section{Introduction: COVID-19 victims}

This work is meant as an illustration of Simpson's paradox, also known as the Yule-Simpson paradox (Yule 1903; Simpson 1951). We use the COVID-19 pandemics as an occasion to show how some basic mathematical observations apply to many aspects of life, in this case victims of the COVID-19 pandemics and scientific contributions of countries as measured by citations per publication.

On June 22, 2020, R.R.'s local Flemish newspaper, De Standaard, mentioned that in any age group men have a higher COVID-19 infection fatality rate (IFR) than women, but in the total population of Belgium women have a higher IFR, see Table 1, as taken from this newspaper article (De Smet 2020). The IFR is the probability that one dies, given that one is infected.

Ronald Rousseau

ronald.rousseau@uantwerpen.be; ronald.rousseau@kuleuven.be

Zhiqi Wang

zhiqi_wang90@126.com

1 WISE Lab, Institute of Science of Science and S\&T Management, Dalian University of Technology, Dalian, People's Republic of China

2 Faculty of Social Sciences, University of Antwerp, Antwerpen, Belgium

3 Dept. MSI, Centre for R\&D Monitoring (ECOOM), KU Leuven, 3000, Leuven, Belgium 
Table 1 Infection fatality rate in different age groups for men and women in Belgium (June 2020)

\begin{tabular}{lcc}
\hline Age groups & Men $(\%)$ & Women $(\%)$ \\
\hline $0-24$ & 0.00 & 0.00 \\
$25-44$ & 0.02 & 0.01 \\
$45-64$ & 0.29 & 0.14 \\
$65-74$ & 2.92 & 1.61 \\
$75-84$ & 5.56 & 3.35 \\
85 and older & 13.20 & 11.07 \\
All ages & 1.18 & 1.31 \\
\hline
\end{tabular}

The fact that in any age group men have a higher infection fatality rate (IFR) than women, but when bringing all age groups together the opposite is the case, seems to be contradictory. This phenomenon is well-known in statistics and is known as Simpson's paradox. Being a quality newspaper, De Standaard also mentioned that the data of Table 1 reflect Simpson's paradox (a term not often used in dailies). In this case, the underlying reason is that there are much more older women than men in Belgium. The reporter got his information from an-yet unpublished - article by Flemish colleagues (Molenberghs et al. 2020). Data shown in Table 1 have been updated recently, but do not detract from the fact that in June 2020 the best available data showed the Yule-Simpson paradox.

\section{The Yule-Simpson paradox}

\section{Simpson's theoretical example}

The Yule-Simpson paradox (Yule 1903; Simpson 1951; Blyth 1972; Gardner 1976) is an expression of a counter-intuitive result that may occur in statistical aggregations. The paradox refers to the fact that outcomes of comparisons between groups are reversed when groups are combined. Real-world examples have been observed in surgery (Charig et al. 1986), clinical trials (Rücker and Schumacher 2008), ecological studies (Allison and Goldberg 2002; Clark et al. 2011) and, citation analysis (Ramanana-Rahary et al. 2009), among others.

Let us consider the following example shown by Simpson (1951). It appears that the two sets of data separately support a certain hypothesis, but, considered together, support the opposite hypothesis. Simpson provided the following fictitious case related to the outcome of a medical treatment (Table 2).

There are 52 cases in total. Among the male population $4 / 7 \approx 0.57$ of the untreated survived, while $8 / 13 \approx 0.62$ of the treated ones did. Hence the treatment had a positive effect among males. Among the females, $2 / 5=0.4$ of the untreated survived, while $12 / 27 \approx 0.44$ of the treated ones did. So, also among the female population, the treatment had a positive effect. However if we consider the whole population (bringing males and females together) we see

Table 2 Simpson's survival data

\begin{tabular}{lrrr}
\hline & \multicolumn{1}{l}{ Male } & \multicolumn{1}{l}{ Female } & \multicolumn{1}{l}{ Total } \\
\hline Untreated & $4 / 7=57 \%$ & $2 / 5=40 \%$ & $6 / 12=50 \%$ \\
Treated & $8 / 13=62 \%$ & $12 / 27=44 \%$ & $20 / 40=50 \%$ \\
\hline
\end{tabular}


that among the untreated ones 6 survived and 6 died and among the treated ones 20 survived and 20 died, pointing at no effect from the treatment.

\section{The general framework}

The Yule-Simpson paradox occurs in the following situation. Three stochastic variables are involved: $X, Y$ and $Z$. In Simpson's example $X$ takes two values: surviving or not surviving; $Y$ also takes two values: being treated or not; and $Z$ represents males or females (these are the ones that are brought together). For the COVID-19 case, $X$ represents dying or not from COVID-19; $Y$ represents males and females and $Z$ represents different age groups (here the age groups are brought together).

Now the Yule-Simpson paradox occurs if the following happens (Blyth, 1972): $X$ takes values $A$ and $A^{\prime}$ (the complement of A); $Y$ takes values $B$ and $B$ ' (the complement of $B$ ); $Z$ takes values $C_{1}, C_{2}, C_{3}, \ldots$ (and if there are only two outcomes possible, we denote them by $C$ and $C^{\prime}$ ).

For all $j=1,2,3, \ldots: P\left(A \mid \mathrm{B}\right.$ and $\left.C_{j}\right)>P\left(A \mid B^{\prime}\right.$ and $\left.C_{j}\right)$

and yet: $P(A \mid B) \leq P\left(A \mid \mathrm{B}^{\prime}\right)$

Here $P(. \mid$.) represents a conditional probability. We also say that the Yule-Simpson paradox occurs if:

For all $j=1,2,3, \ldots: P\left(A \mid \mathrm{B}\right.$ and $\left.C_{j}\right) \geq P\left(A \mid B^{\prime}\right.$ and $\left.C_{j}\right)$

and yet: $P(A \mid B)<P\left(A \mid \mathrm{B}^{\prime}\right)$

Intuitively one might think that as $P(A \mid B)$ is an average of the $P\left(A \mid B\right.$ and $\left.C_{j}\right)$ and similarly for $P\left(A \mid B^{\prime}\right)$ and the $P\left(A \mid B^{\prime}\right.$ and $\left.C_{j}\right)$ the paradox is not possible. Yet, the point is that these averages have different weightings (Blyth 1972). We further note that if $Y$ and $Z$ are independent then the Yule-Simpson paradox is not possible (Blyth 1972).

The Yule-Simpson paradox and its interpretation can be illustrated graphically using socalled median fractions. As we did that already in our previous article (Ramanana-Rahary et al. 2009), published in this journal, we refer the interested reader to that publication.

\section{A short overview of some historical cases of the Yule-Simpson paradox}

As suggested by a reviewer we provide some details on other historical cases of the YuleSimpson paradox.

A well-known historical example relates to tuberculosis deaths in 1910. Referring to Cohen and Nagel (1934, page 449), Wagner (1982) shows that although the overall mortality rate was lower in New York City than in Richmond (VA), the opposite held when data were stratified into whites and non-whites.

One of the best-known examples of the Yule-Simpson paradox is a study of possible gender bias among graduate school admissions in 1973 to the University of California, Berkeley. On the whole, male students were more likely than female ones to be admitted. However, when examining the individual departments, it appeared that six out of 85 departments were significantly biased against male applicants, whereas four were significantly biased against female ones. A detailed study of the data by Bickel et al. (1975) revealed that female students tended to apply to more competitive departments with low rates of admission whereas men tended to apply to less competitive departments with high rates of admission. It was concluded that there was no bias from the side of the university, but a selection bias on the part of the applicants. 
Table 3 Successful removal of kidney stones (Charig et al. 1986)

Table 4 Male line (has or does not have the trait)

Table 5 Female line (has or does not have the trait)

Table 6 Mixed (sum table)

\begin{tabular}{lrrl}
\hline & Large stones & Small stones & \multicolumn{1}{l}{ All } \\
\hline Open surgery & $192 / 263=73 \%$ & $81 / 87=93 \%$ & $273 / 350=78 \%$ \\
$\begin{array}{c}\text { Percutaneous } \\
\text { nephroli- } \\
\text { thotomy }\end{array}$ & $55 / 80=62 \%$ & $234 / 270=83 \%$ & $289 / 350=83 \%$ \\
\hline
\end{tabular}

\begin{tabular}{lll}
\hline & Son has & $\begin{array}{l}\text { Son does } \\
\text { not have }\end{array}$ \\
\hline Father has & 25 & 25 \\
Father does not have & 25 & 25 \\
\hline
\end{tabular}

\begin{tabular}{lll}
\hline & Daughter has & $\begin{array}{l}\text { Daughter } \\
\text { does not } \\
\text { have }\end{array}$ \\
\hline Mother has & 1 & 9 \\
Mother does not have & 9 & 81 \\
\hline
\end{tabular}

\begin{tabular}{lll}
\hline & Offspring has & $\begin{array}{l}\text { Offspring } \\
\text { does not } \\
\text { have }\end{array}$ \\
\hline Parent has & 26 & 34 \\
Parent does not have & 34 & 106 \\
\hline
\end{tabular}

Julious and Mullee (1994) analyzed data obtained by Charig et. al. (1986) on the efficiency of two treatments to remove kidney stones (open surgery vs. percutaneous nephrolithotomy). The new technique proved successful on the whole, but stratification by the size of the kidney stones led to different conclusions. The confounding factor was that surgeons' choice of treatment was not random but influenced by the size of the stone. This example supported the necessity to use random trials.

We next illustrate this example with the real data in the form of a contingency table (Table 3).

The stochastic variable $X$ takes the values successful or not; $Y$ takes the values open surgery or percutaneous nephrolithotomy and $Z$ takes the values large stones or small ones.

Finally, we discuss Yule's original example (Yule 1903, p. 133). This case is related to the study of inheritance and is formulated differently. Yule provides (fictitious) data on a trait that is not hereditary in the male line and neither in the female line, but occurs with a different probability (Tables 4, 5 and 6). Bringing data together in equal proportions suggests inheritance, which is the wrong conclusion.

Whether or not the father has the trait, the probability that his son has it is $25 / 50=50 \%$; whether or not the mother has the trait, the probability that her daughter has it is $1 / 10=9 / 90=10 \%$. Yet making these calculations in the sum table yields $26 / 60=43 \%$ and 
$34 / 140=24 \%$. Yule writes that a large but illusory inheritance is created simply by mixing the two distinct records. He then warns against pooling data about heterogeneous material in general.

Mittal (1991) refers to this form of the paradox as Yule's association paradox, while he refers to the case shown by Simpson (1951) as Yule's reversal paradox (because the signs in the aggregated table are reversed). Mittal (1991) quotes Nagel as the source for attaching the name of Yule to these two types of paradoxes.

\section{An interpretation related to impact}

\section{Direct impact: a fictitious example}

The boxes in Table 7, taken from (Ramanana-Rahary et al. 2009), represent direct impact (citations per publication). Research is performed by two countries in two related disciplines. We add a row for 'All countries' (here the two countries). We see that Country 2 is better than Country 1 in Discipline 1 as well as in Discipline 2. Yet adding the results leads to the opposite conclusion.

\section{Relative impact: an example}

The example above also produces an inversion for relative impacts. If, instead of comparing Country 1 and Country 2, we compare each country separately to their aggregate 'All countries', say 'the World', we see that in the above example: (score country 1) $<$ (Score all countries) in Disciplines 1 and 2, i.e. the relative impact with respect to the world, of Country 1 is inferior to unity, but (Country 1)>(All countries) for All disciplines, i.e. its world relative impact is superior to unity. For Country 2 the opposite holds. Numerical values of relative impacts are given in Table 8.

\section{An abstract framework}

Let us put this in an abstract framework. The Yule-Simpson paradox occurs if Table 9 is given, together with the requirements that $A / U<C / W$ and $B / V<D / X$ while $(A+B) /$ $(U+V) \geq(C+D) /(W+X)$.

Note that $A, B, C, D, U, V, W$ and $X$ are given, not just the numerical values of the fractions. From now on, we assume that the reader understands the Yule-Simpson paradox and hence we will simply refer to it as the Yule-Simpson phenomenon.

We recall from (Ramanana-Rahary et al. 2009) two simple mathematical results, using the general term 'player' instead of 'country'.

Table 7 An example of direct impact

\begin{tabular}{llll}
\hline Direct impact (fractions) & Discipline 1 & Discipline 2 & Total: all disciplines \\
\hline Country 1 & $60 / 100=0.60$ & $1 / 10=0.10$ & $61 / 110=0.55$ \\
Country 2 & $9 / 10=0.90$ & $30 / 100=0.30$ & $39 / 110=0.35$ \\
All countries & $69 / 110=0.63$ & $31 / 110=0.28$ & $100 / 220=0.45$ \\
\hline
\end{tabular}


Table 8 Relative impacts expressed as fractions

\begin{tabular}{llll}
\hline Relative impact & Discipline 1 & Discipline 2 & All disciplines \\
\hline Country 1 & $(60 / 100) /(69 / 110)=0.96$ & $(1 / 10) /(31 / 110)=0.35$ & $(61 / 110) /(100 / 220)=1.22$ \\
Country 2 & $(9 / 10) /(69 / 110)=1.43$ & $(30 / 100) /(31 / 110)=1.06$ & $(39 / 110) /(100 / 220)=0.78$ \\
All countries & $(69 / 110) /(69 / 110)=1.00$ & $(31 / 110) /(31 / 110)=1.00$ & $(100 / 220) /(100 / 220)=1.00$ \\
\hline
\end{tabular}

Table 9 General framework for the Yule-Simpson paradox

\begin{tabular}{llll}
\hline & Discipline 1 & Discipline 2 & All disciplines \\
\hline Player 1 & $A / U$ & $B / V$ & $(A+B) /(U+V)$ \\
Player 2 & $C / W$ & $D / X$ & $(C+D) /(W+X)$ \\
All players & $(A+C) /(U+W)$ & $(B+D) /(V+X)$ & $(A+B+C+D) /$ \\
& & & $(U+V+W+X)$ \\
\hline
\end{tabular}

Proposition 1 The Yule-Simpson phenomenon is present for the pair (Player 1, Player 2) for direct impact, if and only if it is also present for relative impact.

This follows immediately from the fact that if in an equality both sides are multiplied or divided by the same positive number, the inequality stays invariant.

Proposition 2 The Yule-Simpson phenomenon for the pair (Player1, Player2) is present if and only if it is present for the pair (Player1, Both Players).

\section{Real-world citation examples}

\section{The Essential Science Indicators}

The examples we will show are retrieved from the Essential Science Indicators (ESI). Data from the science citation index-expanded (SCIE) and the social sciences citation index (SSCI) in the web of science (WoS) core collection are subdivided into 22 broad fields based on publication and citation performance (Essential Science Indicators 2020). These 22 broad fields are shown in the appendix (Table 16). Data, only articles and reviews, cover a rolling 10 year period and include bimonthly updates. For our investigation, it is important to recall that articles are classified according to the journal in which they are published and that each journal is assigned to only one field. Multidisciplinary journals, however, are an exception to this rule: here a reclassification is performed at the paper level, based on an analysis of the cited references. Data were collected in September 2020. We restricted data to countries that have at least 500 publications (over a 10 year period), except for a few cases where we compared a country with all the other countries in the database, for which the Yule-Simpson phenomenon occurs rarely.

The role of a discipline (as in Table 9) is played by one ESI field. We do not intend to be complete and to combine each ESI field with each other ESI field (it makes little sense to combine, e.g., chemistry with social sciences, general). We just provide some examples in fields for which it may be acceptable to combine them (Tables 10, 11, 12, 13 and 14). 
Table 10 Mathematics-Physics

\begin{tabular}{llrrr}
\hline Pair & Fields & \multicolumn{1}{l}{ Mathematics } & \multicolumn{1}{l}{ Physics } & \multicolumn{1}{l}{ Union of the two fields } \\
\hline \multirow{2}{*}{$\begin{array}{l}\text { Countries/regions } \\
\text { 1 }\end{array}$ India } & $57,531 / 14,412=3.99$ & $629,484 / 62,347=10.10$ & $687,015 / 76,759=8.95$ \\
& China & $499,826 / 98,963=5.05$ & $2,776,267 / 268,479=10.34$ & $3,276,093 / 367,442=8.92$ \\
2 & Spain & $94,778 / 18,510=5.12$ & $788,581 / 40,532=19.46$ & $883,359 / 59,042=14.96$ \\
& Canada & $93,442 / 17,060=5.48$ & $618,910 / 31,724=19.51$ & $712,352 / 48,784=14.60$ \\
3 & Czech Rep & $21,983 / 5265=4.18$ & $232,167 / 13,950=16.64$ & $254,150 / 19,215=13.23$ \\
& South Africa & $15,826 / 3457=4.58$ & $116,993 / 6782=17.25$ & $132,819 / 10,239=12.97$ \\
4 & Thailand & $7,760 / 2,062=3.76$ & $54,882 / 3804=14.43$ & $62,642 / 5866=10.68$ \\
& Romania & $37,077 / 7548=4.91$ & $144,592 / 9611=15.04$ & $181,669 / 17,159=10.59$ \\
5 & Poland & $45,347 / 11,818=3.84$ & $388,627 / 29,717=13.08$ & $433,974 / 41,535=10.45$ \\
& Turkey & $50,640 / 11,103=4.56$ & $220,212 / 16,588=13.28$ & $433,974 / 41,535=9.78$ \\
\hline
\end{tabular}

Table 11 Molecular biology and genetics-Neuroscience and behavior

\begin{tabular}{|c|c|c|c|c|}
\hline Pair & Fields & $\begin{array}{l}\text { Molecular biology and } \\
\text { genetics }\end{array}$ & $\begin{array}{l}\text { Neuroscience and } \\
\text { behavior }\end{array}$ & Union of the two fields \\
\hline & Countries /region & & & \\
\hline \multirow[t]{2}{*}{1} & Singapore & $234,333 / 5471=42.83$ & $61,580 / 3041=20.25$ & $295,913 / 8512=34.76$ \\
\hline & Finland & $216,039 / 4654=46.42$ & $124,023 / 5,153=24.07$ & $340,062 / 9807=34.68$ \\
\hline \multirow[t]{2}{*}{2} & Northern Ireland & $41,464 / 769=53.92$ & $15,953 / 636=25.08$ & $57,417 / 1405=40.87$ \\
\hline & Wales & $96,910 / 1745=55.54$ & $68,065 / 2,512=27.10$ & $164,975 / 4257=38.75$ \\
\hline \multirow[t]{2}{*}{3} & Slovenia & $25,336 / 757=33.47$ & $13,042 / 658=19.82$ & $38,378 / 1415=27.12$ \\
\hline & Australia & $596,389 / 17,538=34.01$ & $516,531 / 24,153=21.39$ & $1,112,920 / 41,691=26.69$ \\
\hline
\end{tabular}

Differences in impact (cites per publication) are often rather small so that one may say that they are not statistically significant. Yet, we do not step into the statistical morass of significance testing (Schneider 2015) and just stick to rankings.

\section{Examples where the Yule-Simpson phenomenon occurs}

Although a rather rare event, we also found examples of the Yule-Simpson phenomenon between a country and all other countries in the database, see Table 15. Here, we included cases with less than 500 publications.

\section{Remarks}

1. We found several more examples involving countries and fields with fewer publications.

2. The cases shown in this contribution are just examples of a phenomenon that might not be well-known to all colleagues. We did not check the 'correctness' of the data in the used database.

3. Countries that are compared have relative citations that do not differ much, although their absolute numbers of publications and citations may differ considerably. As countries are rarely compared in this way, this leads to unexpected 'relatives'. So we see India 
Table 12 Computer science-Mathematics

\begin{tabular}{|c|c|c|c|c|}
\hline Pair & Fields & Computer science & Mathematics & Union of the two fields \\
\hline & Countries / regions & & & \\
\hline \multirow[t]{2}{*}{1} & The Netherlands & $79,050 / 7140=11.07$ & $25,789 / 4508=5.72$ & $104,839 / 11,648=9.00$ \\
\hline & England & $271,827 / 24,125=11.27$ & $130,313 / 20,992=6.21$ & $402,140 / 45,117=8.91$ \\
\hline \multirow[t]{2}{*}{2} & Wales & $15,328 / 1382=11.09$ & $5185 / 864=6.00$ & $20,513 / 2246=9.13$ \\
\hline & Finland & $45,640 / 4087=11.17$ & $17,583 / 2880=6.11$ & $63,223 / 6967=9.07$ \\
\hline \multirow[t]{2}{*}{3} & Malaysia & $40,311 / 4275=9.43$ & $8605 / 1782=4.83$ & $48,916 / 6057=8.08$ \\
\hline & Greece & $52,053 / 5357=9.72$ & $18,302 / 3390=5.40$ & $70,355 / 8747=8.04$ \\
\hline \multirow[t]{2}{*}{4} & Ireland & $24,065 / 2540=9.47$ & $8799 / 1729=5.09$ & $32,864 / 4269=7.70$ \\
\hline & Belgium & $46,543 / 4853=9.59$ & $26,759 / 4733=5.65$ & $73,302 / 9586=7.65$ \\
\hline
\end{tabular}

Table 13 Clinical medicine-Molecular biology and genetics

\begin{tabular}{|c|c|c|c|c|}
\hline Pair & Fields & Clinical Medicine & $\begin{array}{l}\text { Molecular Biology \& } \\
\text { Genetics }\end{array}$ & Union of the two fields \\
\hline & Countries/regions & & & \\
\hline \multirow[t]{2}{*}{1} & USA & $17,699,989 / 926,526=19.10$ & $6,722,408 / 184,228=36.49$ & $24,422,397 / 1,110,754=21.99$ \\
\hline & Greece & $575,770 / 29,343=19.62$ & $98,950 / 2562=38.62$ & $674,720 / 31,905=21.15$ \\
\hline \multirow[t]{2}{*}{2} & Singapore & $389,425 / 19,658=19.81$ & $234,333 / 5471=42.83$ & $623,758 / 25,129=24.82$ \\
\hline & Ireland & $404,061 / 19,041=21.22$ & $137,677 / 3007=45.79$ & $541,738 / 22,048=24.57$ \\
\hline \multirow[t]{2}{*}{3} & Israel & $577,269 / 30,091=19.18$ & $248,119 / 6611=37.53$ & $825,388 / 36,702=22.49$ \\
\hline & Greece & $575,770 / 29,343=19.62$ & $98,950 / 2562=38.62$ & $674,720 / 31,905=21.15$ \\
\hline \multirow[t]{2}{*}{4} & Germany & $4,028,859 / 211,381=19.06$ & $1,474,034 / 44,261=33.30$ & $5,502,893 / 255,642=21.53$ \\
\hline & Greece & $575,770 / 29,343=19.62$ & $98,950 / 2562=38.62$ & $674,720 / 31,905=21.15$ \\
\hline
\end{tabular}

Table 14 Clinical medicine-Biology and biochemistry

\begin{tabular}{llcrr}
\hline Pair & Fields & Clinical medicine & Biology and biochemistry & Union of the two fields \\
\hline \multirow{2}{*}{$\begin{array}{l}\text { Countries /regions } \\
1\end{array}$} & Colombia & $137,736 / 6459=21.32$ & $17,711 / 1583=11.19$ & $155,447 / 8042=19.33$ \\
& Argentina & $238,754 / 10,681=22.35$ & $72,420 / 5,854=12.37$ & $311,174 / 16,535=18.82$ \\
2 & Philippines & $91,065 / 2003=45.46$ & $4,759 / 530=8.98$ & $95,824 / 2533=37.83$ \\
& Ukraine & $79,517 / 1562=50,91$ & $11,264 / 995=11.32$ & $90,781 / 2557=35.50$ \\
3 & Vietnam & $73,058 / 2200=33.21$ & $10,451 / 1039=10.06$ & $83,509 / 3239=25.78$ \\
& Costa Rica & $25,090 / 673=37.28$ & $6021 / 569=10.58$ & $31,111 / 1242=25.05$ \\
4 & Qatar & $76,059 / 3670=20.72$ & $7770 / 630=12.33$ & $83,829 / 4300=19.50$ \\
& Argentina & $238,754 / 10,681=22.35$ & $72,420 / 5854=12.37$ & $311,174 / 16,535=18.82$ \\
\hline
\end{tabular}

and China, the Netherlands and England, Qatar and Argentina and the USA and Greece, to name a few.

4. The ESI categories are disjoint and hence it makes sense to add publications and citations. A similar exercise is not directly possible with WoS categories or SCImago categories. 
Table 15 Comparisons with the world

\begin{tabular}{|c|c|c|c|c|}
\hline Pair & Fields & Molecular biology and genetics & Neuroscience and behavior & Union of the two fields \\
\hline \multirow[t]{2}{*}{1} & Vietnam & $12,590 / 536=23.49$ & $2,896 / 167=17.34$ & $15,486 / 703=22.03$ \\
\hline & All others & $12,098,390 / 500,861=24.16$ & $10,034,241 / 539,131=18.61$ & $22,132,631 / 1,039,992=21.28$ \\
\hline \multirow[t]{3}{*}{2} & Bangladesh & $10,792 / 460=23.46$ & $2,674 / 165=16.21$ & $13,466 / 625=21.55$ \\
\hline & All others & $12,100,188 / 500,937=24.16$ & $10,034,463 / 539,133=18.61$ & $22,134,651 / 104,070=21.28$ \\
\hline & Fields & Computer Science & Mathematics & Union of the two fields \\
\hline \multirow[t]{3}{*}{3} & Taiwan & $121,248 / 14,414=8.41$ & $33,194 / 7,089=4.68$ & $154,442 / 21,503=7.18$ \\
\hline & All others & $3,362,268 / 394,445=8.52$ & $2,162,699 / 451,667=4.79$ & $5,524,967 / 846,112=6.53$ \\
\hline & Fields & Social Sciences, General & Economics \& Business & Union of the two fields \\
\hline \multirow[t]{2}{*}{4} & Ethiopia & $18,742 / 2,389=7.85$ & $1,915 / 193=9.92$ & $20,657 / 2,582=8.00$ \\
\hline & All others & $7,982,499 / 1,021,260=7.82$ & $2,920,014 / 300,898=9.70$ & $10,902,513 / 1,322,158=8.25$ \\
\hline
\end{tabular}

5. Incites uses whole counting and hence when two countries are compared a part of their data overlap. The Yule-Simpson phenomenon between two countries might or might not occur if fractional counting were used. Moreover, assume that one removes all coauthored articles between two countries then again the Yule-Simpson phenomenon may or may not occur. Indeed, inequalities may reverse when removing joint publications and their citations. Let $\mathrm{A} / \mathrm{U}<\mathrm{C} / \mathrm{W}$ as in $400 / 300<500 / 350$. If now these countries have 200 publications with 100 citations in common and these are removed then we have $300 / 100>400 / 150$ with reversed inequality.

\section{Conclusions}

Although the Yule-Simpson phenomenon in citation analysis is not common, it isn't extremely rare either. This is shown in this contribution. It is a phenomenon one should be aware of, otherwise one may encounter unforeseen surprises. Assume, for instance, that it is the scientific aim of a country to do better, citation-wise, than world average in the two related fields $F_{1}$ and $F_{2}$. Then this aim may be reached for the union of the two fields, but for none of the fields separately. Such a possibility is just a mathematical fact. The COVID19 example and the historical examples illustrated that the Yule-Simpson phenomenon may occur in any aspect of life.

From the historical examples, we learned that one can make a distinction between two cases. Sometimes, such as in the Berkeley students case and for the kidney stone case, there is a clear (human) selection procedure at work and it makes no sense to aggregate data. Sometimes, as in the COVID-19 example, there is a natural stratification (age groups), but again it is not important at all to collect information on the aggregated data. So, in general, we think that it is not a good idea to aggregate data as it leads to a clear loss of information.

In the citation analysis presented here, we artificially aggregated fields, yet these fields themselves are aggregates and we did not try to find the relation, e.g., between mathematics and its subfields (algebra, geometry, topology, analysis, etc.). So for citation analysis, the answer to the question "Should one aggregate or not?" depends on the aim of the investigation. 
As an aside we showed that in terms of relative citations, i.e. citations per publication, large, well-known countries such as England and the USA may, in some fields, become comparable with smaller ones such as the Netherlands and Greece.

\section{Appendix}

See Table 16.

Table 16 The 22 broad research fields used in the Essential Science Indicators (ESI)

\begin{tabular}{|c|c|c|c|c|}
\hline No & Research fields & $\begin{array}{l}\text { Web of science } \\
\text { documents }\end{array}$ & Cites & Cites/Paper \\
\hline 1 & Clinical medicine & $2,942,586$ & $39,526,641$ & 13.43 \\
\hline 2 & Chemistry & $1,826,753$ & $29,215,059$ & 15.99 \\
\hline 3 & Materials science & 985,562 & $15,895,770$ & 16.13 \\
\hline 4 & Engineering & $1,491,145$ & $14,079,684$ & 9.44 \\
\hline 5 & Biology and biochemistry & 773,798 & $13,633,446$ & 17.62 \\
\hline 6 & Physics & $1,114,358$ & $13,258,475$ & 11.90 \\
\hline 7 & Molecular biology and genetics & 501,397 & $12,110,980$ & 24.15 \\
\hline 8 & Neuroscience and behavior & 539,298 & $10,037,137$ & 18.61 \\
\hline 9 & Social sciences, General & $1,023,649$ & $8,001,241$ & 7.82 \\
\hline 10 & Environment/Ecology & 583,684 & $7,971,101$ & 13.66 \\
\hline 11 & Plant \& Animal Science & 778,100 & $7,795,841$ & 10.02 \\
\hline 12 & Geosciences & 502,457 & $6,863,716$ & 13.66 \\
\hline 13 & Psychiatry/Psychology & 449,811 & $5,785,883$ & 12.86 \\
\hline 14 & Pharmacology and toxicology & 435,488 & $5,771,696$ & 13.25 \\
\hline 15 & Immunology & 272,454 & $5,255,343$ & 19.29 \\
\hline 16 & Agricultural sciences & 461,003 & $4,683,342$ & 10.16 \\
\hline 17 & Microbiology & 221,551 & $3,581,914$ & 16.17 \\
\hline 18 & Computer science & 408,859 & $3,483,516$ & 8.52 \\
\hline 19 & Economics and business & 301,091 & $2,921,929$ & 9.70 \\
\hline 20 & Space science & 154,642 & $2,913,112$ & 18.84 \\
\hline 21 & Mathematics & 458,756 & $2,195,893$ & 4.79 \\
\hline 22 & Multidisciplinary & 23,406 & 423,822 & 18.11 \\
\hline
\end{tabular}

Note: Data are collected in September 2020.

Acknowledgements The authors thank the reviewers for useful suggestions to improve the original manuscript.

\section{References}

Allison, V. J., \& Goldberg, D. E. (2002). Species-level versus community-level patterns of mycorrhizal dependence on phosphorus: an example of Simpson's paradox. Functional Ecology, 16(3), 346-352. 
Bickel, J. P., Hammel, A. E., \& O'Connell, J. W. (1975). Sex bias in graduate admissions: Data from Berkeley. Science, 187(4175), 398-404.

Blyth, C. R. (1972). On Simpson's paradox and the sure-thing principle. Journal of the American Statistical Association., 67(338), 364-366.

Charig, C. R., Webb, D. R., Payne, S. R., \& Wickham, O. E. (1986). Comparison of treatment of renal calculi by operative surgery, percutaneous nephrolithotomy and extracorporeal shock wave lithotripsy. BMJ, 292(6524), 879-882.

Clark, J. S., Bell, D. M., Hersh, M. H., Kwit, M. C., Moran, E., Salk, C., et al. (2011). Individual-scale variation, species-scale differences: inference needed to understand diversity. Ecology Letters, 14(12), 1273-1287.

Cohen, M. R., \& Nagel, E. (1934). An Introduction to Logic and Scientific Methods. New York: Hartcourt, Brace and World.

De Smet, D. (2020). Is corona erger dan de griep? (Is corona worse than the flu?) De Standaard, 22 June 2020.

Essential Science Indicators. (2020). Essential science indicators. Clarivate analytics. Retrieved from https ://clarivate.com/webofsciencegroup/solutions/essential-science-indicators/. Accessed September 2020.

Gardner, M. (1976). Mathematical games On the fabric of inductive logic and some probability paradoxes. Scientific American, 234(3), 119-124.

Julious, S. A., \& Mullee, M. A. (1994). Confounding and Simpson's paradox. BMJ, 309(6967), 1480-1481.

Mittal, Y. (1991). Homogeneity of subpopulations and Simpson's paradox. Journal of the American Statistical Association, 86(413), 167-172.

Molenberghs, G., Faes, C., Aerts, J., Theeten, H., Devleesschauwer, B., Bustos Sierra, N., et al. (2020). Belgian COVID-19 mortality excess deaths number of deaths per million and infection fatality rates. MedRxiv Preprint Server for Health Sciences. https://doi.org/10.1101/2020.06.20.20136234.

Ramanana-Rahary, S., Zitt, M., \& Rousseau, R. (2009). Aggregation properties of relative impact and other classical indicators: convexity issues and the Yule-Simpson paradox. Scientometrics, 9(2), 311-327.

Rücker, G., \& Schumacher, M. (2008). Simpson's paradox visualized: The example of the Rosiglitazone meta-analysis. BMC Medical Research Methodology, 8, 34.

Schneider, J. W. (2015). Null hypothesis significance tests. A mix-up of two different theories: the basis for widespread confusion and numerous misinterpretations. Scientometrics, 102(1), 411-432.

Simpson, E. H. (1951). The interpretation of interaction in contingency tables. Journal of the Royal Statistical Society Series B, 13(2), 238-241.

Wagner, C. H. (1982). Simpson's paradox in real life. The American Statistician, 36(1), 46-48.

Yule, G. U. (1903). Notes on the theory of association of attributes of statistics. Biometrika, 2(2), 121-134. 